\title{
The Phoenix Deep Survey: Evolution of Star Forming Galaxies
}

\author{
A. M. Hopkins ${ }^{1,2}$, J. Afonso ${ }^{3}$, A. Georgakakis ${ }^{4}$, M. Sullivan ${ }^{5}$, B. Mobasher ${ }^{6}$, \\ and L. E. Cram ${ }^{7}$ \\ 1 University of Pittsburgh, Department of Physics and Astronomy \\ 3941 O'Hara St, Pittsburgh, PA 15206, USA \\ 2 Hubble Fellow; ahopkins@phyast.pitt.edu \\ 3 CAAUL, Observatory of Lisbon, Tapada da Ajuda, 1349-018 Lisbon, Portugal \\ ${ }^{4}$ National Athens Observatory, I.Metaxa \& Vas.Pavlou str., Athens 15236, Greece \\ 5 University of Toronto, 60 St. George St, Toronto, Ontario M5S 3H8, Canada \\ 6 STScI, 3700 San Martin Drive, Baltimore, MD 21218, USA \\ 7 Australian Research Council, GPO Box 2702, Canberra, ACT 2601, Australia
}

\begin{abstract}
The Phoenix Deep Survey (PDS) is a multiwavelength survey based on deep $1.4 \mathrm{GHz}$ radio observations used to identify a large sample of star forming galaxies to $z=1$. Photometric redshifts are estimated for the optical counterparts to the radiodetected galaxies, and their uncertainties quantified by comparison with spectroscopic redshift measurements. The photometric redshift estimates and associated best-fitting spectral energy distributions are used in a stacking analysis exploring the mean radio properties of $U$-band selected galaxies. Average flux densities of a few $\mu$ Jy are measured.
\end{abstract}

\section{Introduction}

The study of galaxy evolution in recent years has included a strong focus on the star formation properties of galaxies. Many of these studies are based primarily on selection at ultraviolet (UV) and optical wavelengths, known to be strongly affected by obscuration due to dust. It has been shown that selection at these wavelengths results in samples of star forming systems that miss a significant fraction of heavily obscured galaxies [20]. There have moreover been suggestions that the most vigorous star forming (SF) systems suffer the most obscuration $[2,17,6,22,16]$. Radio selection provides an efficient tool to construct a SF galaxy sample free from dust induced biases, and the average obscuration in such samples indeed appears significantly higher than in optically selected samples $[2,17]$.

Motivated to construct a homogeneously selected sample of SF galaxies, unbiased by the effects of obscuration due to dust, the Phoenix Deep Survey (PDS, see http://www.atnf.csiro.au/people/ahopkins/phoenix/) is based on a deep $(60 \mu \mathrm{Jy})$, wide-area ( 4.5 square degree) $1.4 \mathrm{GHz}$ survey with the Australia Telescope Compact Array. This provides one of the largest existing deep $1.4 \mathrm{GHz}$ source catalogues [14] containing a large fraction of SF galaxies spanning the broad redshift range $0<z<1$. The PDS has already been highly successful in providing a basis for several investigations of the nature of SF galaxies and their evolution $([12,2,14]$ and references therein). Throughout the present investigation we assume a $\left(\Omega_{M}=0.3, \Omega_{\Lambda}=0.7, H_{0}=70\right)$ cosmology. 


\section{Photometric redshift analysis}

Deep $U B V R I$ observations of about one square degree within the PDS have recently been analysed (these are described in detail in [21]). These data achieve a $5 \sigma$ level of $R_{\mathrm{AB}} \approx 24.5$ and optical catalogues have been constructed and cross-correlated with the $1.4 \mathrm{GHz}$ catalogues. This multicolour data has been used to estimate photometric redshifts for all $\approx 40000$ galaxies detected in each of the 5 bands. This includes about 800 optical counterparts of the radio detected galaxies in this area.

For our analysis we use the photometric redshift code of Connolly et al. [10] (see also [7]). We use SED templates based on those of Coleman et al. [8] (hereafter CWW), providing four standard SEDs (E/S0, Sbc, Scd, Im), extended in the UV and IR wavelength regions using the GISSEL98 code [5]. Rather than using these four SEDs directly, we use the method of optimal subspace filtering [7] to provide a large number (61) of smoothly interpolated SEDs based on the reference CWW SEDs. This supports more realistic type estimates for most of the galaxies. We allow the possible photometric redshifts to range from 0.0 to 1.3 and also apply a prior constraining the absolute magnitudes of the galaxies to the broad range $-29<M_{B}<-16$ (having the effect of removing photometric redshift fits with unphysically high or low redshifts).

Figure 1 compares the photometric redshift estimate with spectroscopic redshift for 116 radio sources with an optical counterpart having both $U B V R I$ detections and spectroscopic data. The filled symbols (including points) indicate the spectroscopic classification, while the open symbols give an estimate of the best-fitting SED template type (after binning the 61 subspace filtered templates into four bins, based approximately on the closest CWW-type template). The reliability of the photometric redshifts can be characterised in several ways. The rms of $|\Delta z|=\left|z_{\text {photo }}-z_{\text {spec }}\right|$ is 0.1 for these 116 galaxies. The rms of $\left|\log \left[\left(1+z_{\text {photo }}\right) /\left(1+z_{\text {spec }}\right)\right]\right|$ is 0.028 (implying a typical uncertainty of $7 \%$ in $\left.1+z_{\text {photo }}\right)$. The rms of $|\Delta z| /\left(1+z_{\text {spec }}\right)$ is 0.065 . This level of reliability in the photometric redshifts compares favourably with that of other analyses $[11,19]$. As well as providing reasonable redshift estimates, the best-fitting SED is also a good indicator of the galaxy type, in the sense that spectroscopic absorptionline systems are mostly well-fit by early-type SEDs, while SF systems are mostly well-fit by late-type SEDs.

\section{Stacking analysis of $U$-band galaxies}

The technique of stacking small subregions of an image at the locations of a known population of objects that are not otherwise detected, in order to extract a rough estimate of the mean emission properties of a population, has been used with some success at X-ray wavelengths $[4,18]$. This technique has been applied to XMM observations of the PDS [12] to explore the X-ray properties of radio-detected SF galaxies. Following this success we extended the method

to radio wavelengths, performing a stacking analysis using the $1.4 \mathrm{GHz}$ mosaic 


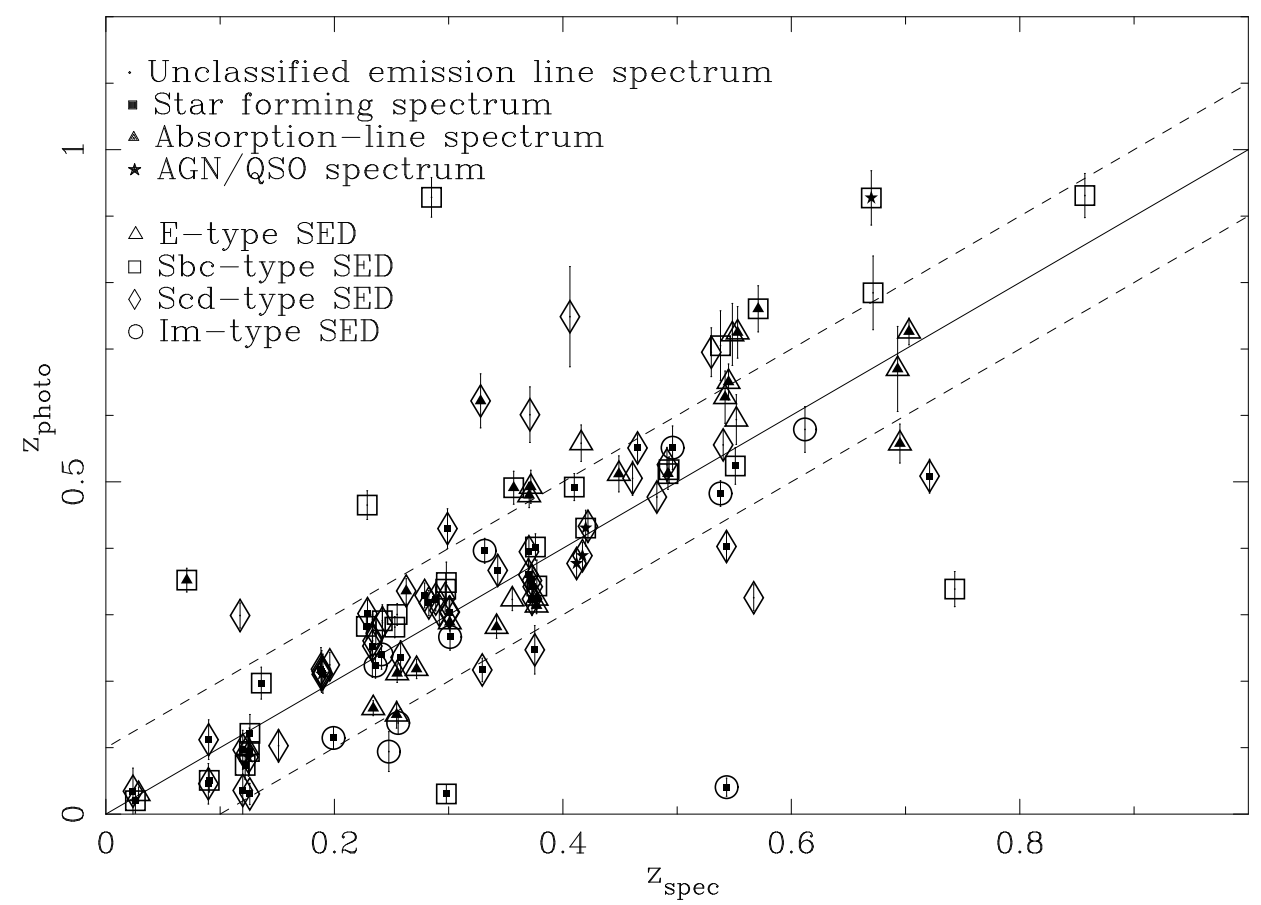

Fig. 1. A comparison of spectroscopic and photometric redshifts for the spectroscopically observed sub-sample. The points and filled symbols refer to the spectroscopic classification of the galaxies [13], while the open symbols refer to the best-fitting SED from the photometric redshift estimation. The open symbols are estimated by binning the 61 subspace filtered SEDs approximately into the closest CWW-type classification. Note that this figure shows redshifts in linear units, with the dashed lines indicating offsets of \pm 0.1 in $z_{\text {photo }}$ about the one-to-one line.

image of the PDS to explore the mean radio properties of extremely red galaxies (ERGs) not otherwise detected at $1.4 \mathrm{GHz}$ [15]. These results have implications for the expected average radio luminosities and inferred star formation rates of ERGs. Further investigation of the radio properties of ERGs through stacking analyses are explored elsewhere in this volume [1]. We now further extend this technique to explore the average $1.4 \mathrm{GHz}$ properties of a population of $U$-band selected galaxies, which is expected to include a large fraction of SF galaxies. This will provide an estimate of the typical radio properties for the population of "normal" or quiescent SF galaxies, as opposed to the starbursts that often dominate studies of star formation in galaxies.

The available $U$-band data reaches a $5 \sigma$ detection limit of $U_{\mathrm{AB}} \approx 25.0$. To explore radio flux density trends with both redshift and galaxy type, we take advantage of the photometric redshifts and best-fitting SED types to split the 40000 galaxies into three bins in redshift and three bins in SED type (described as "early," "mid" and "late," the CWW types Sbc and Scd being included in 
A. M. Hopkins et al.

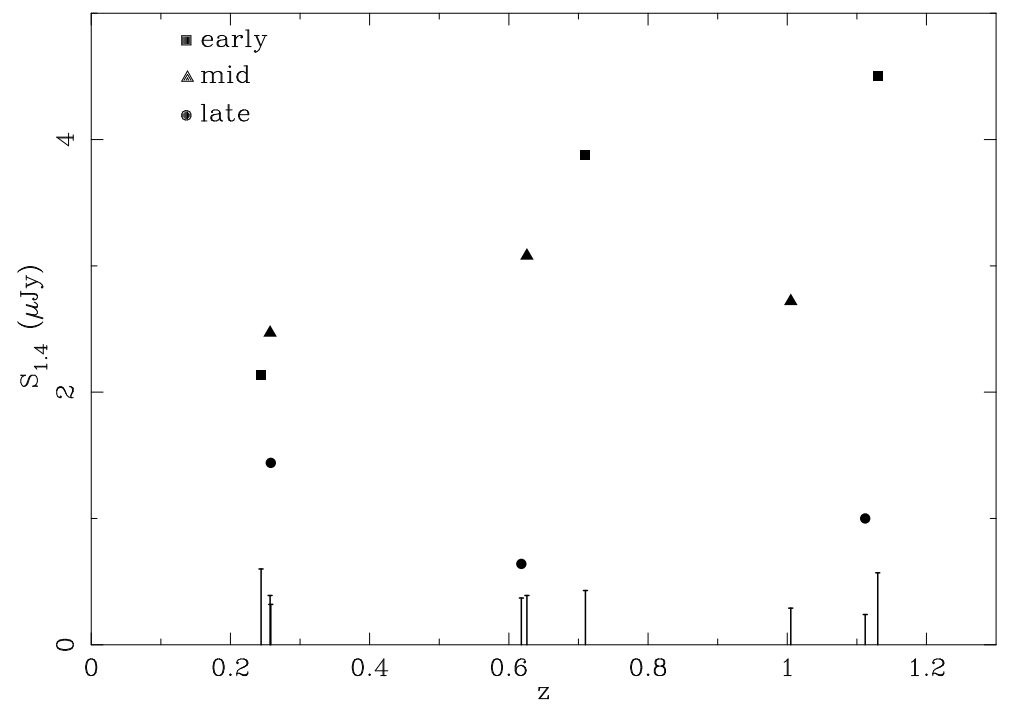

Fig. 2. Average $1.4 \mathrm{GHz}$ flux density inferred from the stacking analysis for galaxies of early (squares), mid (triangles) and late (circles) SED type. The flux densities are shown as a function of the median photometric redshift of all the objects contributing to each of the nine stacked images. The vertical bars below each point indicate the rms noise level of the stacked image in which each measurement was made, with detections ranging from $3.6-9 \sigma$. The only non-detection is the late-type SED (circle) in the middle redshift bin, and is shown to indicate the upper limit for this stacked image (an arrow was omitted to avoid clutter with the rms noise level bars).

the "mid" type). The numbers of galaxies in each bin are given in Table 1 . For the stacking analysis a subregion of $2^{\prime}$ square was extracted from the PDS $1.4 \mathrm{GHz}$ image at the location of each of the $U$-band galaxies. To ensure that radio detections or uncatalogued low signal-to-noise $(\mathrm{S} / \mathrm{N})$ radio emission do not bias the stacking signal, subregions are excluded from the stacking analysis if the average $1.4 \mathrm{GHz}$ emission in a $14^{\prime \prime} \times 14^{\prime \prime}$ region centred at the location of the $U$-band galaxy is above some $\mathrm{S} / \mathrm{N}$ threshold. The threshold chosen was a fairly conservative $1.5 \sigma$, although the results change only marginally if slightly higher thresholds $(2-3 \sigma)$ are used. Since the noise level is not uniform across the PDS $1.4 \mathrm{GHz}$ image [14], the individual subregions are weighted by the inverse square of the rms noise background during the averaging step, in order to maximise the $\mathrm{S} / \mathrm{N}$ of the resulting stacked image.

The results of the stacking analysis are shown in Figures 2 and 3. Of the nine stacked images constructed, eight show confident detections $(>3.6 \sigma)$. The exception is the middle redshift bin for the late-type SED systems, where the peak flux at the expected location of the source is $1.7 \sigma$. The measured $1.4 \mathrm{GHz}$ flux densities for the stacking results are shown in Figure 2 as a function of the median photometric redshift for the objects contributing to each final stacked 


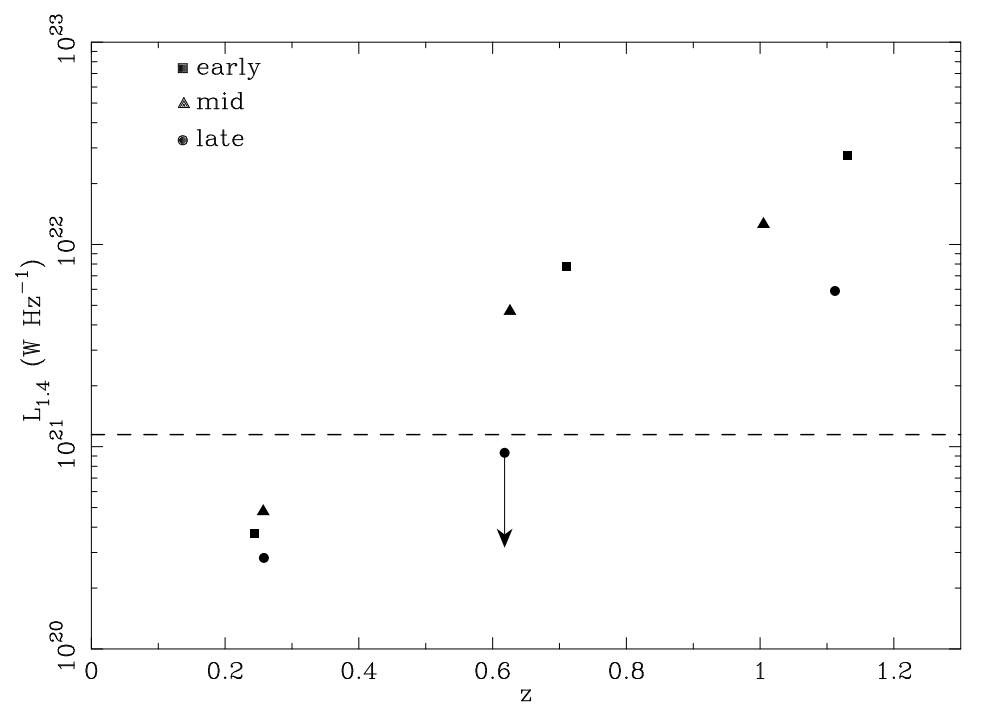

Fig. 3. Average $1.4 \mathrm{GHz}$ luminosity inferred from the flux density measured in the stacking analysis, and the median photometric redshift. The non-detection is now indicated as an upper limit. The dashed line indicates the luminosity corresponding to an SFR of $1 M_{\odot} \mathrm{yr}^{-1}$ using the calibration of [3].

image. The measured flux densities are of order a few $\mu \mathrm{Jy}$ for each of the stacked detections, in rms backgrounds around 0.3 to $0.5 \mu \mathrm{Jy}$. The "mid"-type SED class seem to predominantly show flux densities between the "early" and "late" types, consistent with what might be expected if it was comprised of a combination of both active galactic nuclei (AGNs) and SF systems, or of systems driven by both processes.

The luminosities derived from these flux densities and the median photometric redshifts are shown as a function of photometric redshift in Figure 3. The trend to higher luminosities at higher redshifts for all SED types is likely to be a result of our magnitude-limited selection, since at high redshifts only the higher ( $U$-band) luminosity systems are being sampled, and the radio luminosity is likely to be correlated at some level with the $U$-band luminosity [17]. The dashed line in this figure indicates a star formation rate (SFR) of $1 M_{\odot} \mathrm{yr}^{-1}$ using the calibration of [3] (this line is about $40 \%$ higher than if the calibration of [9] were used). This shows that through the use of the stacking technique it is possible to develop some insight into the $1.4 \mathrm{GHz}$ properties of "normal" or quiescent SF galaxies. The fact that early type SED systems are detected at similar flux densities may suggest either that low luminosity AGN are present in significant numbers in normal galaxies, or that early type galaxies can support these low levels of star formation, or even (since the flux density in early types seems consistently higher than in late types) that both processes might be occurring in these systems. 
Table 1. Numbers of galaxies in redshift and type bins for the $U$-band stacking

\begin{tabular}{lccc}
\hline Redshift range & Early & Mid & Late \\
\hline $0.00<z<0.43$ & 1358 & 3349 & 6377 \\
$0.43<z<0.87$ & 3349 & 4009 & 3384 \\
$0.87<z<1.30$ & 1562 & 6910 & 8663 \\
\hline
\end{tabular}

AMH acknowledges support provided by NASA through Hubble Fellowship grant HST-HF-01140.01-A awarded by STScI. JA acknowledges the support from the Science and Technology Foundation (FCT, Portugal) through the fellowship BPD-5535-2001 and the research grant POCTI-FNU-43805-2001.

\section{References}

1. J. Afonso, A. Hopkins, B. Mobasher, L. Cram, A. Georgakakis, M. Sullivan: This Proceedings (2003)

2. J. Afonso, A. Hopkins, B. Mobasher, C. Almeida: ApJ 597, 269 (2003)

3. E. F. Bell: ApJ 586, 794 (2003)

4. W. N. Brandt, et al.: AJ 122, 1 (2001)

5. A. G. Bruzual, S. Charlot: ApJ, 405, 538 (1993)

6. V. Buat, A. Boselli, G. Gavazzi, C. Bonfanti: A\&A 383, 801 (2002)

7. T. Budavári, A. S. Szalay, A. J. Connolly, I. Csabai, M. Dickinson: AJ 120, 1588 (2000)

8. G. D. Coleman, C.-C. Wu, D. W. Weedman: ApJS 43, 393 (1980)

9. J. J. Condon: ARA\&A 30, 575 (1992)

10. A. J. Connolly, I. Csabai, A. S. Szalay, D. C. Koo, R. G. Kron, J. A. Munn: AJ 110, 2655 (1995)

11. A. Fernández-Soto, K. M. Lanzetta, A. Yahil: ApJ 513, 34 (1999)

12. A. Georgakakis, A. M. Hopkins, M. Sullivan, J. Afonso, I. Georgantopoulos, B. Mobasher, L. E. Cram: MNRAS 345, 939 (2003)

13. A. Georgakakis, B. Mobasher, L. Cram, A. Hopkins, C. Lidman, M. RowanRobinson: MNRAS 306, 708 (1999)

14. A. M. Hopkins, J. Afonso, B. Chan, L. E. Cram, A. Georgakakis, B. Mobasher: AJ 125, $465(2003)$

15. A. M. Hopkins, J. Afonso, A. Georgakakis, M. Sullivan, B. Mobasher, L. E. Cram: 'Extremely red galaxies in the Phoenix Deep Survey'. In: Multiwavelength Cosmology, (in press; astro-ph/0309147)

16. A. M. Hopkins, A. J. Connolly, D. B. Haarsma, L. E. Cram: AJ 122, 288 (2001)

17. A. M. Hopkins, et al.: ApJ (2003) (in press; astro-ph/0306621)

18. K. Nandra, R. F. Mushotzky, K. Arnaud, C. C. Steidel, K. L. Adelberger, J. P. Gardner, H. I. Teplitz, R. A. Windhorst: ApJ 576, 625 (2002)

19. M. Rowan-Robinson: MNRAS 345, 819 (2003)

20. I. Smail, F. N. Owen, G. E. Morrison, W. C. Keel, R. J. Ivison, M. J. Ledlow: ApJ 581, 844 (2002)

21. M. Sullivan, A. M. Hopkins, J. Afonso, A. Georgakakis, B. Chan, L. E. Cram, B. Mobasher: (in preparation)

22. M. Sullivan, B. Mobasher, B. Chan, L. Cram, R. Ellis, M. Treyer, A. Hopkins: ApJ 558, $72(2001)$ 